\title{
Del documento digital a la biblioteca virtual
}

\author{
Blanca Rodríguez Bravo \\ Lourdes Santos de Paz \\ Universidad de León
}

\section{1. Resumen}

Se reflexiona sobre las distintas denominaciones que han recibido las bibliotecas y los documentos en el momento presente como consecuencia del uso de la tecnología: documentos electrónicos, digitales o virtuales; y bibliotecas electrónicas, digitales, híbridas, virtuales . Se constata que las evoluciones de las bibliotecas y los documentos son paralelas, pues la existencia de documentos digitalesvirtuales permite la biblioteca digital-virtual. Se reflexiona sobre los cambios sucedidos en las bibliotecas con la aparición y desarrollo de los documentos digitales como parte de su colección. Se analiza la evolución en el último cuarto del siglo XX y la situación a la que en ellas nos enfrentamos. La evolución producida en las bibliotecas a partir del uso de tecnologías ha repercutido en distintos aspectos: funcionalidad, servicios, productos, etc. Se intenta precisar los distintos momentos por los que ha transcurrido la rápida evolución de estos últimos años y valorar si existe correspondencia con la terminología utilizada para nombrar a la "nueva biblioteca" en la bibliografía especializada. Se constata la poca delimitación referida a los conceptos que expresan términos como biblioteca electrónica, digital, virtual o híbrida, y la necesidad de clarificarlos. (Autores)

Palabras clave: Biblioteca electrónica. Biblioteca digital. Biblioteca virtual. Biblioteca híbrida. Documento electrónico. Documento digital.

\section{2. Abstract}

Reflection on the different denominations that libraries and documents are receiving in the present moment as a consequence of technological innovations: electronic, digital or virtual documents; and electronic, digital, hybrid or virtual libraries. Both evolutions - librariesan and documents - are parallel, because it is the existence of digital-virtual documents that allows the digital-virtual library. The changes that have experienced the libraries with the appearance and development of the digital documents as part of their collection are analysed. The evolution that libraries have experienced in the last decades are considered. Such

Scire. $8: 2$ (jul.-dic. 2002) 43-52. 
evolution has influenced decisively their functionality, services, products, etc. The different moments of this evolution will be analised, toguether with the terminology used in the specialized bibliography. Concepts expressed through terms like electronic, digital, virtual or hybrid library are used in a very broad sense, so they need a futher clarification. (Authors)

Keywords: Electronic library. Digital library. Virtual library. Hybrid library. Electronic document. Digital document.

\section{Introducción}

El objetivo de este trabajo es reflexionar sobre la evolución reciente y el estado actual de las bibliotecas y de su materia prima, los documentos. Para ello, reflexionaremos sobre las distintas denominaciones que unas y otros han experimentado, y sobre los conceptos que se derivan de ellas: documentos electrónicos, digitales o informáticos y bibliotecas electrónicas, digitales, híbridas o virtuales. Constataremos que ambas evoluciones (bibliotecas-documentos) son paralelas pues la existencia de documentos digitales, una de cuyas principales características es su virtualidad, permite la biblioteca digital-virtual. Ambos adjetivos se complementan, ya que, si digital hace referencia a la composición o estructura de los documentos, y por extensión a las bibliotecas que los contienen, virtual se refiere a la accesibilidad documental, y con respecto a las bibliotecas se aplica a aquellas que ofertan servicios a distancia.

Pretendemos reflexionar sobre los cambios que ha supuesto para las bibliotecas la aparición y desarrollo de los documentos digitales como parte de la colección, su parte virtual. Se está pasando de una situación en que la información mayoritariamente se expresaba mediante formatos analógicos a otra en la que el formato digital va acaparando cotas importantes en el mercado de la industria de los contenidos y, como ponen de relieve Agustín y Muñoz (1997), en un proceso documental en el que toman parte documentos digitales, no sólo cambia el soporte, también lo hacen el canal, el código y la organización de la información. Los documentos digitales generan una nueva manera de gestionar y transmitir la información y, por lo tanto, provocan cambios fundamentales en las bibliotecas.

El origen de este cambio puede situarse en la introducción de la informatización en el sistema de la biblioteca. Desde los años cincuenta, en los que se introdujo la aplicación del ordenador para realizar búsquedas bibliográficas, hasta el momento presente, la evolución de los trabajos y servicios en la biblioteca se ha llevado a cabo de manera casi paralela al avance sucedido en el ámbito propio de las tecnologías. Desde la inclusión de tecnologías usadas como herramientas liberadoras de tareas mecánicas, hasta el uso de las tecnologías de la información 
actuales ha habido una transición al principio más lenta y en los últimos años muy acelerada, que está dando un vuelco a los sistemas tradicionales de biblioteca.

La situación actual de las bibliotecas en las que conviven documentos tradicionales - analógicos - y novedosos — digitales - junto con servicios presenciales y a distancia — virtuales — recibe distintas denominaciones: bibliotecas sin paredes, bibliotecas sin papeles, bibliotecas electrónicas, bibliotecas digitales, bibliotecas virtuales, bibliotecas híbridas. A nuestro parecer no hay muchas divergencias de significado, sólo difiere el punto de enfoque: si pensamos en los servicios, hablaremos de bibliotecas virtuales o sin paredes; si pensamos en los recursos, hablaremos de bibliotecas sin papeles, electrónicas o digitales. Estas denominaciones pueden servir, según algunos autores, para marcar un proceso evolutivo, aunque se suelen intercambiar en el uso que la literatura profesional hace de ellas. Como señala Moreiro (1) realmente nuestras bibliotecas son mayoritariamente híbridas, en ellas conviven los documentos y los servicios tradicionales con los nuevos.

Hechas estas precisiones creemos que se pueden diferenciar varios estadios en la evolución de las bibliotecas, y para denominarlos vamos a utilizar los adjetivos electrónica, digital y virtual, para concluir con que nuestras bibliotecas en realidad son híbridas y que quizá esa situación perdure, pues sea la más favorable para la generalidad de los usuarios. Saorín, García y Martínez (1998) establecen tres estadios evolutivos que denominan Escenario 0, Escenario 1 y Escenario 2. El escenario 0 equivaldría a la situación evolutiva de las bibliotecas electrónicas, el 1 a las bibliotecas digitales o híbridas hoy predominantes y el 2 a las bibliotecas virtuales.

\section{La biblioteca electrónica}

En puridad, como señala Codina (2000), los adjetivos electrónico y digital se utilizan como sinónimos sin serlo. Ambos adjetivos unidos al sustantivo bibliotecas hacen referencia a los documentos que componen su fondo. Por ello conviene aclarar el significado de estos términos. Un documento electrónico es aquel que precisa de una máquina que funcione de forma electrónica, sea analógica o digital, para ser reproducido o visualizado. Un documento digital o un documento informático sería aquel documento electrónico que está codificado sobre la base de la codificación binaria, y que precisa de un ordenador para leerlo. El concepto de documento digital es más restringido que el concepto de documento electrónico, que lo engloba. Por ejemplo, una cinta de vídeo es un documento electrónico pero no es digital sino analógico.

Parece que el uso dominante de la expresión bibliotecas electrónicas se ha hecho en referencia a un primer estadio de evolución. Según Rowley (1998) es a

Scire. $8: 2$ (jul.-dic. 2002) 43-52. 
partir de este primer uso de la tecnología cuando podemos hablar de biblioteca electrónica, denominación que relaciona ordenadores y bibliotecas. Creemos que este término resulta adecuado para distinguir las bibliotecas que comienzan a automatizar algunas tareas, apoyándose en ordenadores. Estas bibliotecas tienen contenidos en soporte electrónico y el acceso es por medios físicos, utilizando tecnología. Ontalba (2000) sitúa a esta biblioteca en una primera fase de aplicación de las tecnologías de la información en los procesos documentales. Da acceso local a información en formato electrónico, aunque no intervienen elementos digitales ni telemáticos. Lo que la caracteriza es la automatización de algunos procesos, fundamentalmente la catalogación. La consulta a un módulo OPAC de un sistema integrado de gestión bibliotecaria es común denominador y elemento básico de los servicios de estas bibliotecas. Se desarrolla entre los años 60 y 80 .

\section{La biblioteca digital}

Será el proceso de desarrollo tecnológico el que variará el concepto y dará paso a la biblioteca denominada digital. Este término se usa fundamentalmente para referirse a los centros que utilizan las tecnologías de la información para mejorar sus servicios, pero, y esto es lo que distingue a esta biblioteca de la electrónica inicial, por hacerlo a distancia, a través de redes telemáticas gracias a las posibilidades que ofrece el nuevo documento: el documento digital. La biblioteca digital es consecuencia del uso intensivo de las tecnologías de la información y de las telecomunicaciones. Tiene su desarrollo en la década de los noventa, de manera paralela al desarrollo del documento digital y a la implantación de Internet. El documento digital ha supuesto la enfatización y priorización de uno de sus componentes, el mensaje, y la minusvaloración del otro, el soporte. Ahora se habla más de recursos que de documentos. La expresión "recursos informativos" está relacionada con dos de las características de los comienzos de siglo que vivimos: la importancia de la tecnología y el valor económico concedido al tándem información-conocimiento, conceptuado como un bien más.

La organización de una biblioteca destinada a proporcionar el acceso a la información electrónica genera cambios con respecto al proceso documental tradicional y al acceso, ya que se modifica la manera de transmitir la información. La biblioteca digital se convierte en centro de transmisión de la información tomando parte activa tanto en la gestión y diseminación como en la creación de la información. La biblioteca se convierte en productora de documentos, de guías de recursos y otros productos destinados al usuario, dada la facilidad actual para publicar en la web. En la biblioteca digital se advierte la idea de pérdida del carácter patrimonialista propio de la biblioteca tradicional. Ahora la información se alquila en vez de comprarla. El proceso de selección de la información cobra importancia destacada, pues la información de calidad resulta cara. Cobrará

Scire. $8: 2$ (jul.-dic. 2002) 43-52. 
mayor relieve el just in time con respecto del just in case. La biblioteca será una gestora de licencias que permitan al usuario acceder a la información electrónica. Los nuevos recursos de información no están bajo el control físico del bibliotecario. El sistema de distribución varía pues a veces estos fondos están instalados en un servidor del productor o del distribuidor y otras veces en un servidor local administrado por la propia biblioteca. Los distribuidores suministran los recursos dotados de la correspondiente organización y del sistema de recuperación necesario para ser usados. A la biblioteca le queda la tarea de integrarlos con el resto de instrumentos de recuperación propios.

Los catálogos de referencias serán ahora guías de recursos de documentos de acceso remoto que pueden adoptar la forma de documentos electrónicos hipertextuales organizados por materias y dispuestos en una página web. Los documentos a texto completo que sean muy demandados pueden almacenarse en un disco óptico para ahorrar costes. Asimismo podrá incorporarse a la colección el resultado de algunas búsquedas realizadas por el usuario en su navegación libre y que se estimen interesantes. De esta manera, éste se convierte en parte activa de la selección como si de una desiderata se tratara. Será muy necesario un mantenimiento continuo que pase por la aplicación de una evaluación rigurosa de la calidad de los recursos, así como por una conservación electrónica tangible, referida a pretender una continua funcionalidad del sistema que garantice un acceso a largo plazo.

El tratamiento documental para la información no presencial en nuestro fondo, que se encuentra alojada en lugares ajenos, pero que se quiere referenciar porque se ajusta a las necesidades de la colección, requiere atención. La descripción se basa en el uso de metadatos que facilitan la identificación de los distintos elementos y los hacen recuperables. Estos lenguajes han dado el relevo al sistema ISBD proponiendo una serie de elementos de información mediante los que deben codificar los documentos sus propios productores: Dublín Core, Text Encoding Iniciative son algunos ejemplos. A esto hay que añadir el resurgir de los sistemas clasificatorios presentados ahora como una herramienta útil para organizar los recursos en Internet. La organización de la información en objetos físicos se reemplaza por una más flexible en objetos lógicos y el acceso físico y único a la colección se reemplaza por una organización múltiple de objetos de información (Ortiz-Repiso: 2001). El acceso múltiple plantea cuestiones legales relativas a los derechos de autor y licencias de utilización. En un entorno digital el concepto del copyright es diferente en la medida en que el control de las copias desaparece. La mayoría de los recursos digitales son muy fáciles de reproducir y son potencialmente accesibles por múltiples usuarios al mismo tiempo.

En cuanto a los servicios, hay que decir que se desarrollan en detrimento de los procesos que son compartidos. No obstante, aparece una necesidad de diseñar técnicas para obtener información relevante y esto hará que no desaparezca la

Scire. $8: 2$ (jul.-dic. 2002) 43-52. 
actuación del bibliotecario o del gestor de la información. Aunque la hipertextualidad de los documentos web y la amigabilidad de las interfaces de catálogos y bases de datos facilitan mucho al usuario la búsqueda de información, no es previsible la desintermediación ni la autosuficiencia del usuario, dada la complejidad creciente del mundo de la información. El bibliotecario deberá familiarizarse con nuevas técnicas de acceso al ingente crecimiento cuantitativo y cualitativo de los recursos digitales. Tendrá que estar capacitado para diseñar, crear y mantener instrumentos que permitan identificar, localizar, organizar y difundir la información que el usuario requiere.

Es en el desarrollo de los servicios digitales donde se aprecia el mayor cambio con respecto a lo tradicional debido sobre todo a la naturaleza (digital) de los recursos y al modo de acceso remoto (virtual). A la biblioteca le compete el papel de guía en esta operación creando un servidor que contenga información particular que facilite el acceso al catálogo automatizado, a bases de datos, a documentos electrónicos en texto completo y a todo tipo de herramientas de búsqueda de información. Por medio de páginas web la biblioteca puede organizar directorios de recursos electrónicos organizados por materias y con una breve descripción del contenido. La biblioteca debe hacer accesibles sus servicios al usuario desde cualquier ubicación. No hay necesidad de desplazarse físicamente a la biblioteca, a través de la web, y a través de interfaces con un ordenador se podrán utilizar los servicios que la biblioteca ofrece. La biblioteca pasa así de ser estática a ser distribuida, virtual.

Desde finales de los ochenta y principios de los noventa se viene observando un desplazamiento en la dedicación de las bibliotecas. Se ha pasado de constituir depósitos de información a poner todo el interés en la difusión y la cooperación. No importa tanto el tamaño de la colección como la facilidad de acceso a la información. La biblioteca cada vez más es la intermediaria entre el usuario y la información. Algunos proyectos subvencionados con fondos europeos estudian desarrollos de los servicios bibliotecarios en este entorno: BIBDEL-Libraries Without Walls: The delivery of Library Services to distant users (estudio para la mejora de la disponibilidad y accesibilidad de la información para el usuario remoto aprovechando las posibilidades de diversas tecnologías y recursos) (Abadal, 2000).

Concluyendo, en sintonía con la idea que expone Tennant (1999) sobre la biblioteca digital, los documentos digitales son los que realmente conforman la base y la existencia de la biblioteca digital y hacen que digitales sean sus servicios. Define la biblioteca digital como aquella biblioteca formada por documentos y servicios digitales. Los documentos digitales son unidades que se almacenan, procesan y transfieren por vía digital (binaria). Los servicios digitales son servicios (tales como el de referencia) que son proporcionados digitalmente por medio de ordenadores, a través de redes telemáticas. Precisamente es este último aspecto, el de los servicios, el que hace que aparezca en escena otra denominación para la 
nueva biblioteca: la biblioteca virtual. Si la denominaciones de biblioteca electrónica y de biblioteca digital hacían referencia al tipo de documentos que contienen, la denominación de biblioteca virtual se refiere a los servicios que ofrece.

\section{La biblioteca virtual}

La biblioteca virtual lo es en cuánto a los servicios remotos que ofrece y que existen gracias al desarrollo tecnológico de las bibliotecas, fundamentalmente a la conexión a redes, y a los documentos digitales cuyos contenidos son transmisibles a través de dichas redes. Se le conoce también como "biblioteca sin paredes". Se corresponde con el Escenario 2 de Saorín, García y Martínez (1998). A diferencia de la biblioteca digital, en la que conviven los documentos y los servicios tradicionales con los nuevos, la biblioteca virtual supone la suplantación de la biblioteca física, pues ofrece unicamente servicios remotos.

La virtualidad o accesibilidad a distancia es permitida por la disociación que se produce en el documento digital entre el soporte y el contenido. Según Schamber (1996) el documento digital se caracteriza por ser fácilmente manipulable, enlazable interna y externamente, rápidamente transformable, inherentemente accesible, instantáneamente transportable e infinitamente replicable. Estas características se refieren a los contenidos, los soportes han perdido relevancia, de hecho los mensajes que contienen se copian con facilidad en otro soporte, lo que permite que sean accesibles virtualmente.

Existen ya muestras de bibliotecas virtuales (como por ejemplo la de la UOC (2) o la Miguel de Cervantes (3), cuyos contenidos son vehiculados en soportes digitales únicamente, aunque gran parte de esos contenidos han nacido unidos a otros soportes y han sufrido un proceso de digitalización para integrarse en esas colecciones virtuales. Estas bibliotecas serán cada vez más numerosas, pero junto a ellas creemos que permanecerán potenciadas las bibliotecas digitales, que propiamente deberían ser denominadas híbridas, pues en ellas convivirán los documentos analógicos y los digitales y los servicios presenciales y a distancia.

Dudamos de la desaparición de las bibliotecas físicas porque dudamos de la desaparición de los documentos analógicos, con soportes tangibles, y fundamentalmente de aquéllos en papel. Como dice Codina (2000), al igual que los documentos audiovisuales no han sustituido a la letra impresa porque no cumplen las mismas funciones ni satisfacen las mismas necesidades de información, tampoco los documentos digitales reemplazarán fácilmente a los documentos en soporte papel. Por un lado, el documento digital está fortaleciendo la posición de los documentos textuales en general, pues el código verbal continua siendo el vehículo privilegiado de conservación y acceso a la cultura. Por otro, la accesibilidad o impacto directo del papel, su estética y ergonomía, juegan, hoy por hoy, a favor del libro.

Scire. $8: 2$ (jul.-dic. 2002) 43-52. 


\section{La biblioteca híbrida}

La biblioteca actual que se ajusta a las necesidades presentes es realmente una biblioteca híbrida, aunque la denominación más habitual sea la de digital. Algunos autores (Oppenhein, Pinfield, Rusbridge y también Moreiro y Torres Vargas) la definen destacando su característica más precisa como es la convivencia de documentos tradicionales y documentos digitales. La biblioteca híbrida tiene ante sí el reto de alentar al usuario a conseguir la mejor fuente de información para responder a sus necesidades sin importar el soporte; y a proporcionársela, pues dispondrá de acceso a todos los recursos. La biblioteca híbrida tiene que integrar las nuevas tecnologías junto al sistema tradicional. Esta integración es necesaria, pues no se debe pretender el desmantelamiento del sistema de uso presencial y su sustitución por uno totalmente virtual. Esta labor se presenta como un reto para los bibliotecarios, que tendrán que integrar los recursos electrónicos para dar servicios de calidad, sentando las bases para hacer efectivo el derecho a la información. $¿$ De que forma? Facilitando el acceso: Por ejemplo, proponiendo que el uso de terminales con acceso a Internet se haga de manera natural desde la sala de lectura como al resto de los fondos, y con total gratuidad y libertad de acceso.

La prioridad para la nueva biblioteca será facilitar el acceso a los documentos en cualquier soporte, para ello debe crear una superestructura, una biblioteca digital-virtual, que sirva para compartir recursos. Según Aguillo (2000), la biblioteca híbrida se presenta como la necesaria evolución que debe suceder entre la biblioteca física y su conversión en biblioteca digital-virtual. Otros autores, entre ellos Rowley (1998), muestran su desconfianza de que en un futuro próximo exista una biblioteca digital totalmente pura como único y óptimo acceso a la información, y consideran que la biblioteca seguirá siendo multimedia, disponiendo de los soportes que más se adecuen a las necesidades de su comunidad.

Consideramos que sería conveniente que las bibliotecas híbridas o digitales, integradoras de todo tipo de soportes y servicios, convivan con otras virtuales que dediquen sus presupuestos a la digitalización masiva de documentos analógicos y permitan acceder a los contenidos a los usuarios que viven alejados de los núcleos de población principales. A comienzos del siglo XXI, y con las facilidades que Internet proporciona, no se puede seguir fomentando la existencia de ciudadanos de primera y de segunda, informativamente hablando.

\section{Notas}

(1) En la lección pronunciada en el acto de homenaje al Prof. Martín Fuertes (Universidad de León, marzo de 2001).

(2) Biblioteca UOC: http: //biblio. uoc. es: 443/

(3) Biblioteca Virtual Miguel de Cervantes: http: //cervantesvirtual. com/

Scire. $8: 2$ (jul.-dic. 2002) 43-52. 


\section{Referencias}

Abadal Falgueras, E. (2000). Las bibliotecas en el entorno digital. // Rovira, C. ; Codina, Ll. (dir. ). Documentación digital 2000. Barcelona: UPF, 2000. 202-228. URL: http://docdigital.upf.es.

Aguillo, I. (2000). Bibliotecas "sin paredes": construyendo las nuevas bibliotecas digitales virtuales. // XI Jornadas Bibliotecarias de Andalucía. Sevilla: Asociación Andaluza de Bibliotecarios, 2000. 143-148.

Agustín, C. ; Muñoz, M. (1997). Nuevos usuarios, nuevos documentos. // Scire. 3:1 (enejun. 1997) 75-86.

Anglada i Ferrer, L. M. (2000). Biblioteca digital ¿Mejor, peor o sólo distinta? // Anales de documentación. 3 (2000) 25-39.

Biblioteques digitals i dipòsits nacionals de recursos digitals: seminari organizat per l'Escola Universitaria de Biblioteconomía i Documentació de la Universitat de Barcelona, 12-13 de març de 1999. Barcelona: Universitat de Barcelona, Facultat de Biblioteconomia i Documentació, 2000.

Caraco, A. dir. (2000). Integrer les ressources d'Inernet dans la collection. Villeurbanne: Ecole Nationale Superieure des Sciences de l'Information et des Bibliotheques, 2000.

Caridad Sebastián, M. (coord.) (1999). La sociedad de la información: política, tecnología e industria de los contenidos. Madrid: Centro de Estudios Ramón Areces, 1999.

Codina, L. (2000). El libro digital y la www. Madrid: Tauro, 2000.

Esteban Navarro, M. A. (1998). La biblioteca pública y el derecho de acceso a las redes y los servicios de información electrónica. // Educación y biblioteca. 93 (1998) 22-28.

Faba Pérez, C. ; Moya Anegón. F. de. (1999). Bibliotecas digitales: concepto y principales proyectos. // Investigación Bibliotecológica. 13:26 (1999) 64-78.

Lloret Romero, N. (1999). Cómo plantear un proyecto para el desarrollo de una biblioteca digital // $2^{\text {a }}$ Jornadas Andaluzas de Documentación, 1999. [CD-ROM].

Moscoso, P. (1998). Reflexiones en torno al concepto "recurso de información". // Revista General de Información y Documentación. 8:1 (1998) 327-341.

Ontalba y Ruipérez, J. A. (2000). Bibliotecas digitales españolas: análisis de las primeras realidades // VII Jornadas Españolas de Documentación. Bilbao: Universidad del País Vasco, 2000. 399-409.

Oppenhein, Ch. ; Smithson, D. (1999) What is the hybrid library?. // Journal of Information Science, 25:2 (1999) 97-112.

Ortiz-Repiso Jiménez. (2001) Tecnología de la información en las bibliotecas: inventando el futuro. // V Congreso ISKO-España[CD-ROM]. Alcalá de Henares: Universidad de Alcalá de Henares, 2001.

Pérez Alarcón, A. (1999). La biblioteca digital // Annuari de Biblioteconomia i Documentació: BIBLIODOC 1999. Barcelona: COBDC, 1999. 75-88.

Pinfield, S. Hybrids and clumps. URL: <http://ariadne.ac.uk/issue18/main/> Consultado: 21/7/2001.

Rowley, J. (1998). The electronic library. London: Library Association, 1998.

Scire. $8: 2$ (jul.-dic. 2002) 43-52. 
Rusbridge, Ch. (1998). Towards the hybrid library. [En línea], D-Lib magazine, jul/august 1998. URL: <http: //www. dlib. org/dlib/july98/rusbridge.html>. Consultado: 3/08/2001.

Saorín Pérez, T. ; García Gómez, J. C. ; Martínez Méndez, F. J. (1998). Ideas previas al planteamiento de una metodología para el aprovechamiento de las redes de comunicaciones en las bibliotecas: salas de consulta digitales // X Jornadas Bibliotecarias de Andalucía. Jerez de la Frontera: Asociación Andaluza de Bibliotecarios, 1998. 319-332.

Schamber, L. (1996). What's a document?: Rethinking the concept in uneasy times. // Journal of the American Society for Information Science. 47:9 (1996) 669-671.

Tennant, R. (1999). Digital v. Electronic v. Virtual libraries. Berkeley Digital Library SunSite. UC Regents. URL: 〈http://sunsite.berkeley.edu.mydefinitions.html〉. Consultado: 14/03/2000.

Tochtermann, K. A firts step toward communication in the virtual libraries. URL: <http: //www.cdsl.tamu.edu/cdsl/publs/Klaus/TecRepKlaus.html>. Consultado: 3/08/2001.

Torres Vargas, G. A. (1999). La biblioteca híbrida: ¿un paso hacia el acceso universal a la información?. // Investigación Bibliotecológica. 13: 27 (1999) 142-152. 